\title{
Sistema Inteligente para la Generación Automática de Contratos en el marco de la Ley de Contrataciones Públicas
}

\author{
Rivas, Francklin ${ }^{1,2,3}$; Asprino, Marilena ${ }^{3,4}$; Sarache, Juan' León, Francisco ${ }^{1}$ \\ ${ }^{1}$ Univesidad de Los Andes, Coordinación del Rectorado, Mérida, Venezuela \\ ${ }^{2}$ Universidad Técnica Federico Santa María, Departamento de Informática, Valparaíso, Chile \\ ${ }^{3}$ Pontificia Universidad Católica del Ecuador Sede Ibarra, Escuela de Ingeniería y Jurisprudencia, Ibarra, Ecuador \\ ${ }^{4}$ Univesidad de Los Andes, Facultad de Ciencias Jurídicas y Políticas, Mérida, Venezuela
}

\begin{abstract}
Resumen: En este artículo se presenta el diseño de un sistema inteligente para la creación automática de contratos en el marco de la Ley de Contrataciones Públicas de Venezuela. El sistema está ajustado a la normativa vigente y considera elementos tales como el objeto del contrato, las partes otorgantes, los montos, los aportes sociales requeridos y las cláusulas que debe obligatoriamente contener cada contrato. Como aplicación se desarrolló un sistema web para su utilización en la Universidad de Los Andes, en Venezuela.
\end{abstract}

Palabras clave: Sistemas Inteligentes, Derecho Administrativo, Ley de Contrataciones Públicas, Gobierno Electrónico.

\section{Intelligent System for the Contracts Automatic Generation within the framework of the Public Procurement Law}

\begin{abstract}
This article presents the design of an intelligent system for the automatic creation of contracts within the framework of the Public Procurement Law of Venezuela. The system is adjusted to current regulations and considers elements such as the object of the contract, the granting parties, the amounts, the social contributions required and the clauses that each contract must necessarily contain. As an application, a web system was developed for use at the Universidad de Los Andes, in Venezuela.
\end{abstract}

Keywords: Intelligent Systems, Administrative Law, Public Procurement Law, Electronic Government.

\section{INTRODUCCIÓN}

En Venezuela, la Ley de Contrataciones Públicas de 2008 (Presidencia de la República Bolivariana de Venezuela, 2008) superó la distinción clásica entre el contrato administrativo y el contrato de la Administración, a través del desarrollo de una regulación unitaria del contrato público tanto en lo que respecta al procedimiento de selección de contratistas, como al contenido y ejecución de los contratos de obra, de adquisición de bienes muebles y de prestación de servicios comerciales por ella regulados (Hernández, 2008), de manera que a partir de su entrada en vigencia, todo contrato celebrado por los órganos y entes públicos queda sujeto a ese régimen uniforme desarrollado por la Ley de Contrataciones Públicas, reformada parcialmente en el año 2010 y derogada por el Decreto con Rango, Valor y Fuerza de Ley de Contrataciones Públicas de 13 de noviembre de 2014, (Presidencia de la República Bolivariana de Venezuela, 2014).

La Ley del 2014 dictada por el Ejecutivo Nacional según indica en su Exposición de Motivos, fue creada para propulsar la transformación del sistema económico, estando dentro de sus objetivos "potenciar el aparato productivo nacional, actualizándolo tecnológicamente y articulándolo al nuevo modelo"; e incluye una serie de elementos fundamentales que deben ser considerados en los contratos suscritos por cualquier organismo público. La misma Exposición de Motivos nos revela su importancia, en los términos siguientes: " $L a$ Contratación Pública constituye un importante porcentaje del Producto Interno Bruto Nacional, donde la participación del Estado y los particulares en sus relaciones de intercambio, impulsan el desarrollo económico de la Nación" (Presidencia de la República Bolivariana de Venezuela, 2014).

Esta visión utilitarista del legislador venezolano de la contratación pública coincide con la de la Organización Mundial del Comercio (n.d.), para quien:

La contratación pública tiene una considerable importancia económica tanto a nivel nacional como internacional, y representa una proporción significativa del PIB nacional. A nivel nacional, la contratación de bienes y servicios por organismos gubernamentales proporciona los insumos necesarios que permiten a los gobiernos suministrar servicios públicos y desempeñar otras tareas. Los sistemas de contratación pública repercuten de forma significativa en el uso eficiente de los fondos públicos y, en términos más generales, en la confianza pública en los gobiernos y en la gobernanza. El logro de una buena relación entre calidad y precio, el acceso público a la información relativa a los contratos 
públicos, y las oportunidades equitativas para que los proveedores compitan con miras a obtener contratos públicos, son requisitos esenciales de un sistema eficiente de contratación pública.

Dada su relevancia, la correcta generación de los contratos públicos es una prioridad dentro de la gestión pública, por lo que se hace necesario contar con los conocimientos y la experticia necesaria para evitar incurrir en errores que pudieran tener graves consecuencias desde el punto de vista de la legalidad de las actuaciones, la transparencia del proceso y la confiabilidad en las instituciones del Estado.

En este sentido, se cree que la incorporación de las nuevas tecnologías y el aprovechamiento de sus ventajas y beneficios en el ámbito de la contratación pública, puede ser sumamente favorable para dar respuesta a la creciente exigencia social y normativa de transparencia en la ejecución de todos los procesos de gestión pública, así como para viabilizar la propuesta hecha por algunos expertos de convertir a la contratación en el principal instrumento estratégico para la implantación de políticas públicas (Delgado, 2019).

Las nuevas tecnologías -entre las que se incluyen los sistemas inteligentes- (Aguilar y Rivas, 2001) han sido ampliamente utilizadas en diversos sectores de la vida profesional con gran éxito (Bravo et al., 2014), dado que en ellas se hallan incorporados los elementos distintivos de la inteligencia humana, dentro de los que pueden destacarse, la experticia, criterios, buenas prácticas, análisis y apoyo en la toma de decisiones, entre otros (Rivas y Arciniegas, 2016).

Dentro del amplio espectro de entes públicos, la Universidad de Los Andes (Universidad de Los Andes, n.d.) es una universidad nacional autónoma, financiada por el Estado venezolano, con sede en los tres Estados andinos (Mérida, Táchira y Trujillo) constituyéndose en una de las instituciones de educación superior más importantes del país. Dentro de su organigrama de dependencias se encuentra adscrito al Rectorado la Dirección de los Servicios Jurídicos, órgano de consulta y asesoría técnica y jurídica en la creación y posterior revisión de los diversos documentos y contratos legales que deben ser suscritos por los órganos universitarios investidos de tal competencia, por cuanto le corresponde: "Analizar los procedimientos judiciales o administrativos que pudieran afectar los derechos, bienes intereses directos o indirectos de la Universidad" (Servicio Jurídico de la Universidad de Los Andes, n.d.).

Con el fin de agilizar los procesos, sin sacrificar la calidad de los mismos y resguardando los fines y objetivos públicos que los orientan, se ha desarrollado a través de la presente investigación, la propuesta de diseñar un Sistema Inteligente para la generación automática de los contratos comunmente suscritos por la Universidad de Los Andes, en el marco de la Ley de Contrataciones Públicas, a fin de apoyar a los abogados del Servicio Jurídico en la elaboración de los mismos mediante la inclusión inteligente de los elementos fundamentales que debe contener cada contrato de acuerdo a sus características particulares y de la generación automática de una versión completa del mismo.
La importancia de este trabajo viene dada por la relevancia de la contratación pública para la gestión de los asuntos universitarios, la satisfacción de las necesidades de la comunidad y el cumplimiento de los objetivos de la institución, siempre que se haga de manera oportuna, adecuada y eficaz, bajo criterios de eficiencia en el manejo de los fondos públicos, transparencia y confiabilidad (Organización Mundial de Comercio, 2019). En este sentido, el aporte concreto de la propuesta investigativa consiste en dotar a los abogados del Servicio Jurídico de la Universidad de Los Andes de una herramienta tecnológica de esta naturaleza, para la mejora integral del funcionamiento del mismo, configurando un punto de encuentro entre los Sistemas Inteligentes y el Derecho.

\section{CONTRATOS Y LEY DE CONTRATACIONES PÚBLICAS}

El contrato según establece el Código Civil Venezolano (Congreso de la República de Venezuela, 1982) en su artículo 1.133 es: "una convención entre dos o más personas para constituir, reglar, transmitir, modificar o extinguir entre ellas un vínculo jurídico".

Los contratos forman parte del Derecho de Obligaciones, el cual es definido por Rodríguez-Ferrara (2002) como:

.. la rama del Derecho Privado que estudia la relación obligatoria, vista desde tres ángulos distintos. En primer lugar, se estudia la relación obligatoria en sí, esto es el concepto de relación obligatoria. En segundo lugar, se estudian las fuentes de la relación obligatoria (hechos, circunstancias, normas que generan la relación obligatoria). Se trata aquí de analizar qué hechos o circunstancias dan nacimiento a la relación obligatoria. En tercer lugar, se estudian los efectos de las obligaciones. Se trata de ver qué sucede después que la relación obligatoria ha nacido. En este punto la disyuntiva de la relación obligatoria es precisa: cumplimiento o incumplimiento de la relación obligatoria. El cumplimiento, de ser exacto, conlleva a la extinción de la relación obligatoria. El incumplimiento, por su parte, puede tomar variados caminos de efectos diversos. En principio, aunque no necesariamente, puede conllevar a la responsabilidad del deudor. Pero también puede suceder lo contrario: que el incumplimiento no acarree responsabilidad alguna para el acreedor.

Las condiciones requeridas para la existencia de un contrato están establecidas en el artículo 1.441 del Código Civil Venezolano (Congreso de la República de Venezuela, 1982) y son:

$1^{\circ}$. Consentimiento de las partes;

$2^{\circ}$. Objeto que pueda ser materia de contrato; y 


\section{$3^{\circ}$. Causa lícita.}

Según indica García (2012), los tres elementos que deben coexistir en una relación obligatoria son:

- Elemento Subjetivo: Los sujetos de la relación obligatoria y compuesta por el deudor y acreedor.

- Elemento Objetivo: Es la parte asociada al contenido de la obligación y que es valorada o es susceptible de ser valorada económicamente.

- Elemento jurídico: Es el vínculo entre el deudor y el acreedor y que comprende la actividad o conducta que el primero se compromete a realizar en beneficio del segundo.

Todos estos elementos deben ser considerados al momento de realizar un contrato entre partes para garantizar su validez y el correcto cumplimiento de la finalidad para la cual fue suscrito dicho contrato.

La Administración Pública es definida por Asprino (2016) de las dos maneras siguientes: "La primera, como la actividad desarrollada por las autoridades administrativas para satisfacer las necesidades de la población y, la segunda, como los órganos y entes encargados de llevar a cabo tal actividad o tarea". En este sentido, considerando lo que establece el Código Civil Venezolano en su artículo 1.159: "Los contratos tienen fuerza de Ley entre las partes. No pueden revocarse sino por mutuo consentimiento o por las causas autorizadas por la Ley.".

Con estas consideraciones, es creado el Decreto con Rango, Valor y Fuerza de Ley de Contrataciones Públicas (Presidencia de la República Bolivariana de Venezuela, 2014), el cual tiene como objeto, según indica en su artículo 1:

\begin{abstract}
... regular la actividad del Estado para la adquisición de bienes, prestación de servicios y ejecución de obras, con la finalidad de preservar el patrimonio público, fortalecer la soberanía, desarrollar la capacidad productiva y asegurar la transparencia de las actuaciones de los contratantes sujetos al presente Decreto con Rango, Valor y Fuerza de Ley, coadyuvando al crecimiento sostenido y diversificado de la economía.
\end{abstract}

La Universidad de Los Andes (Universidad de Los Andes, n.d.), formando parte de la Administración Pública y estando regulada por esa ley, debe cuidar que todos los contratos que suscriba cumplan con los requisitos de ley y garantice el correcto manejo por parte de las instancias técnicas y legales de la universidad. Por esa razón, se propone el diseño de un sistema basado en inteligencia artificial que ayude en la creación automática de los contratos que serán suscritos por la Universidad de Los Andes, unificando criterios, formatos y contenidos de los contratos y minimizando las posibilidades de errores en la creación de ellos.

\section{INTELIGENCIA ARTIFICIAL Y SISTEMAS EXPERTOS}

El hombre desde hace mucho tiempo se ha dedicado al estudio de los seres humanos de manera tal de comprender mejor su funcionamiento y tratar de emularlo; creándose técnicas como la robótica encargada de emular las capacidades motoras de los humanos, y la inteligencia artificial encargada de simular el raciocinio, toma de decisiones, posibilidades de representación y aprendizaje (Stuart y Norvig, 2009; Aguilar y Rivas, 2001). Los sistemas expertos (Hidalgo, 1997; García Serrano, 2012; Benchimol et al., 1988) tratan de concentrar en ambientes computacionales el conocimiento adquirido sobre algún entorno particular y cuyo conocimiento proviene de algún experto; brindando la posibilidad de tener el conocimiento del experto en varios lugares simultáneamente y a la vez retener la información que él maneja en caso de su ausencia.

El ser consciente tras la búsqueda de liberarse de tareas pesadas y complejas, empezó a buscar la forma de crear estructuras que emulen tanto el funcionamiento como el raciocinio del ser humano, surgiendo así ciencias encargadas de la parte funcional de cuerpo y la inteligencia artificial encargada del estudio y simulación de las actividades intelectuales del hombre, tales como la manipulación, razonamiento, percepción, aprendizaje, creación, entre otros. Las bondades que poseen los sistemas expertos en función de la capacidad de recopilar la información que posee un experto humano sobre algún entorno en particular y poder sustituirlo en caso de ausencia; los han hecho de gran interés para las industrias en las cuales existe personal altamente calificado que en cualquier momento puede dejar la empresa y dejar un vacío en las funciones que realizaban.

\subsection{Sistemas Basados en Conocimiento}

Es una aplicación informática que soluciona problemas complicados o específicos que de otra manera exigirían ampliamente la pericia humana. Para lograr esto se simula el proceso de razonamiento humano mediante la aplicación de conocimientos e inferencia.

Los sistemas basados en conocimientos (Akerkar y Sajja, 2009) recopilan la información existente sobre algún dominio específico; y si dicha información procede de algún experto en el área, entonces se habla de un sistema experto.

El proceso de adquisición del conocimiento y estructurarlo adecuadamente se conoce con el nombre de Ingeniería de conocimiento.

\section{DISEÑO Y DESARROLLO DE SISTEMA INTELIGENTE PARA LA CREACIÓN AUTOMÁTICA DE LOS CONTRATOS}

Para la creación automática de los contratos de la Universidad de Los Andes en el marco del Decreto con Rango, Valor y Fuerza de Ley de Contrataciones Públicas se diseñó un Sistema Inteligente, particularmente un Sistema Basado en Conocimiento. Para el diseño, se utilizó la metodología presentada en (Rivas, Colina y Rivas, 1998) y utilizada en 
(Rivas-Pérez, Dos Santos, Pardo y Rivas-Echeverría, 2013), la cual consta de seis etapas:

- $\quad$ Etapa 1: Análisis y descripción del problema.

- Etapa 2: Especificación de requerimientos.

- Etapa 3: Análisis de costos, tiempo y recursos.

- Etapa 4: Ingeniería del Conocimiento.

- Etapa 5: Diseño preliminar del Sistema Experto.

- Etapa 6: Desarrollo e Implantación del Sistema Experto.

A continuación, se presenta una descripción de cada una de las etapas desarrolladas:

\section{Etapa 1: Análisis y descripción del problema}

El principal problema conseguido en la revisión de los diferentes contratos suscritos por la ULA son las grandes diferencias que existen entre ellos, así sean del mismo tipo de contratación y de objetos similares. Por otro lado, al existir muchos criterios que deben considerarse en la elaboración del contrato y que depende de elementos variables como el valor de la Unidad Tributaria, el monto establecido a los aportes sociales de las empresas, el monto de la delegación interorgánica otorgada por el Consejo Universitario al Coordinador del Rectorado para la firma de contratos, entre otros, hace que haya elementos susceptibles de cometerse errores involuntarios en la elaboración de los contratos. La creación de un sistema que automáticamente genere un primer borrador de los contratos puede evitar sustancialmente la ocurrencia de errores, permite unificar los criterios y formatos de los contratos y permite tener una base de datos con la información básica y fundamental asociada a los representantes de la Universidad de Los Andes, las empresas y los abogados encargados de la creación de cada contrato.

\section{Etapa 2: Especificación de requerimientos}

En esta etapa se determinan los principales requerimientos que deben ser considerados para el diseño del sistema. Entre los más importantes se pueden destacar:

- Debe ser una aplicación web para que los abogados usuarios del sistema puedan acceder al sistema desde cualquier sitio físico en que se encuentren a través del acceso a un sitio web que estará alojado en un servidor en el edificio central del Rectorado de la Universidad de Los Andes.

- Para efectos de seguridad y trazabilidad del sistema, deben generarse usuarios, quienes serán los que podrán acceder al sistema.

- El sistema debe tener varios módulos:

- Módulo de administración en el cual se permita crear y eliminar los usuarios del sistema. Igualmente debe permitir la modificación de los datos de los representantes de la Universidad de Los Andes (Rector y Coordinador General del Rectorado). Debe permitir introducir los datos de las empresas y además guardarlos para que en siguientes oportunidades en los cuales haya contratos con una misma empresa, los datos ya se encuentren previamente cargados al sistema. Se debe poder incorporar los datos de la designación de los representantes de la Universidad de Los Andes. Por último, debe poderse modificar los datos asociados a los aspectos fiscales del contrato como son el valor en Bolívares de las Unidades Tributarias y el monto del Impuesto al Valor Agregado (IVA) en caso de que se modifiquen cualquiera de ellos.

- $\quad$ Debe poseer un módulo en el cual se pueda proceder a la creación de los contratos y también consultar alguno de los previamente creados.

- El sistema debe permitir definir el tipo de contrato que se va a realizar según lo establecido en el Decreto con Rango, Valor y Fuerza de Ley de Contrataciones Públicas y que son: Adquisición de Bienes, Prestación de Servicios y Ejecución de Obras.

- El sistema debe permitir la generación automática de los contratos en un formato no modificable (.pdf) y que se registre el nombre del abogado responsable de la generación del contrato.

- Debe tratarse de generar algunas plantillas genéricas para los tipos de contratos a ser creados para efectos de unificación de criterios, formatos y datos manejados.

\section{Etapa 3: Análisis de costos, tiempo y recursos.}

De acuerdo a las características del sistema a ser diseñado, se puede determinar preliminarmente que el desarrollo e implementación del mismo en la plataforma de la Universidad de Los Andes puede requerir de un tiempo estimado de seis meses, utilizando como recursos un abogado para la revisión de los modelos de contratos a ser utilizados, un Ingeniero de Conocimiento experto en sistemas inteligentes para la elicitación y estructuración del conocimiento y un Ingeniero de Sistemas, experto en desarrollo de aplicaciones web.

\section{Etapa 4: Ingeniería del Conocimiento:}

En esta etapa se procede a la recolección y organización de la información suministrada por el abogado experto en el área de contratos de Servicio Jurídico de la Universidad de Los Andes, revisión de los diferentes tipos de contratos manejados y presentación de propuestas de modelos genéricos de los contratos a ser generados. Estos modelos genéricos deben contemplar todos los aspectos que sean requeridos en cada tipo de contratos, unificando los formatos, clausulas y contenidos.

\section{Etapa 5: Diseño preliminar del Sistema Experto:}

En esta etapa se procede a la concreción de la organización de la información y a la creación de los formatos genéricos para los diversos tipos de contratos a ser creados por el sistema automático. Se unificó los encabezados de todos los contratos y el número de cláusulas de cada tipo de contratos. El Sistema determina según los montos y según las características del contrato automáticamente la información que debe ser suministrada por el usuario. El sistema se diseña para ser 
implantado en un ambiente web para efecto de accesibilidad e independencia del sistema operativo o equipo a ser utilizado.

\section{Etapa 6: Desarrollo e Implantación del Sistema Experto}

En esta etapa, se desarrolló la aplicación web para la creación de los contratos que suscribirá la Universidad de Los Andes en el marco de la Ley de Contrataciones Públicas. En la Figura 1 se puede observar la pantalla de inicio del sistema, donde un usuario registrado deberá iniciar su sesión en el sistema para utilizarlo.

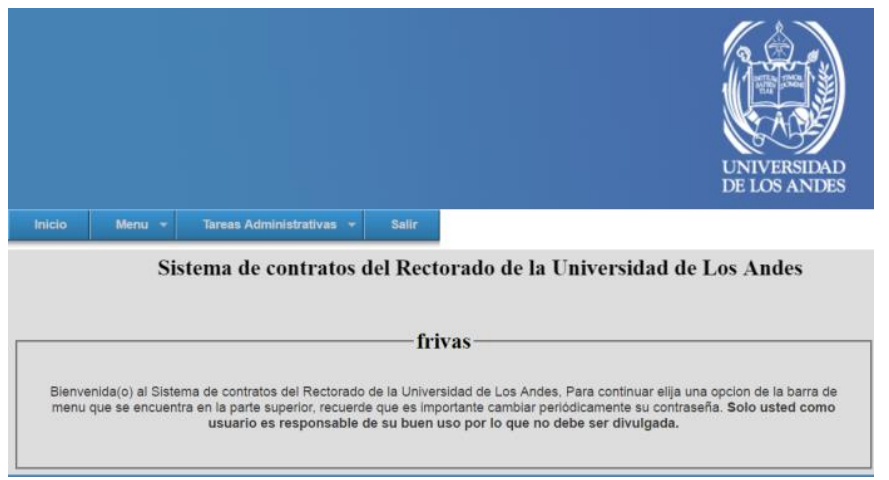

Figura 1. Pantalla de Inicio del Sistema.

En la Figura 2, se puede observar el módulo de gestión de usuarios. Allí se crean los usuarios que podrán tener acceso al sistema y el tipo de privilegio de acceso que puede ser como “administrador" y poder modificar todos los parámetros del sistema o "usuario", los cuales pueden crear contratos, pero no modificar los parámetros fijos del sistema.

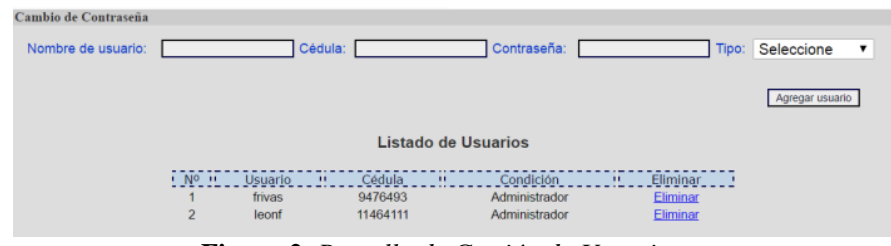

Figura 2. Pantalla de Gestión de Usuarios.

La Figura 3 ilustra el módulo donde se incorpora los datos de los representantes de la Universidad de Los Andes en la firma de los contratos, que son el Rector y el Coordinador del Rectorado.

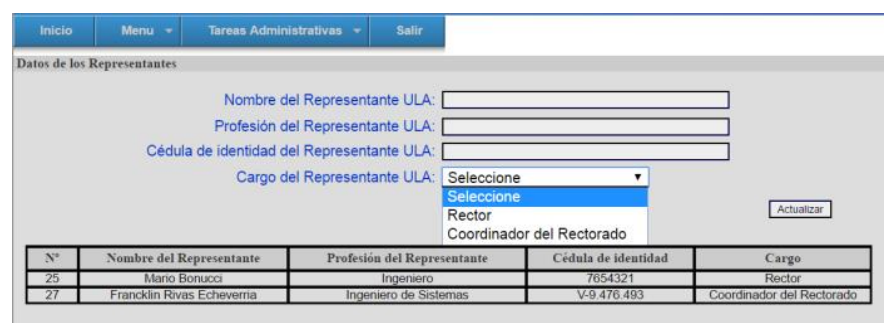

Figura 3. Pantalla de Datos de Representantes de la Universidad.

La Figura 4 ilustra el formato a través del cual cada una de las empresas contratistas es registrada en el sistema, incluyendo los datos que se requerirán para la generación del contrato.

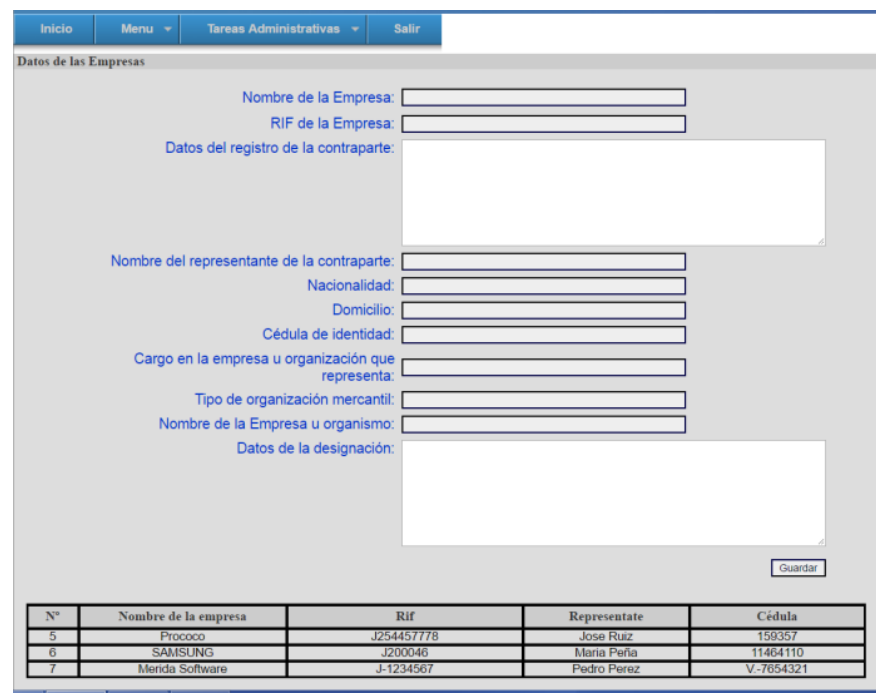

Figura 4. Pantalla de Datos de las empresas.

En la Figura 5 se describen los detalles de las personas autorizadas para la firma de los contratos según la designación y según los montos establecidos en la delegación otorgada por el Consejo Universitario.

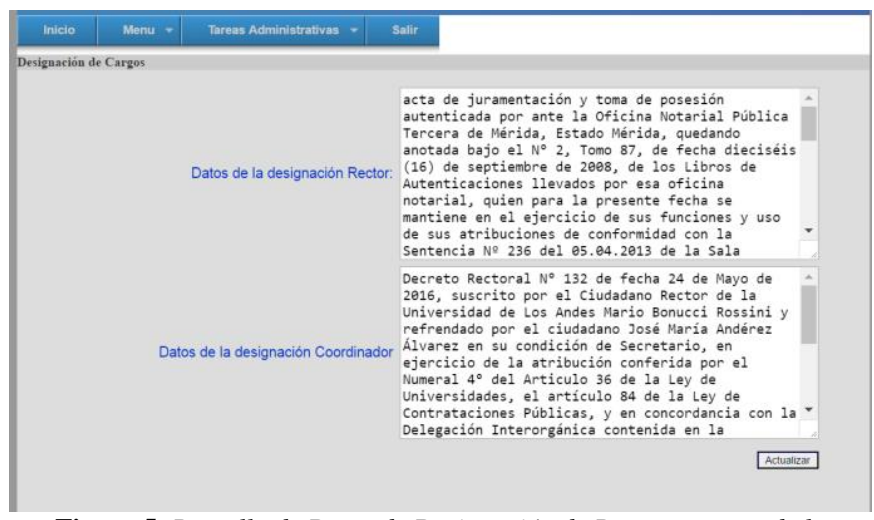

Figura 5. Pantalla de Datos de Designación de Representantes de la Universidad.

La Figura 6 contiene la información relacionada al valor de la unidad tributaria y al porcentaje establecido para el Impuesto al Valor Agregado.

Las Figuras 7 y 8 son las asociadas a la creación de los contratos, en la primera de ellas se selecciona el tipo de contratos según lo establecido en el Decreto con Rango, Valor y Fuerza de Ley de Contrataciones Públicas y en la segunda se incorporan los detalles del contrato a ser suscrito.

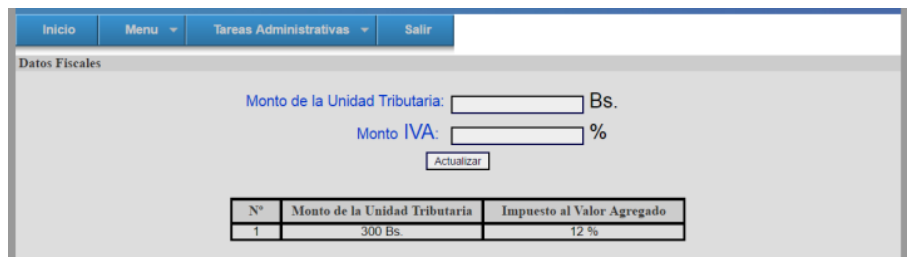

Figura 6. Pantalla de Datos de Información Fiscal. 


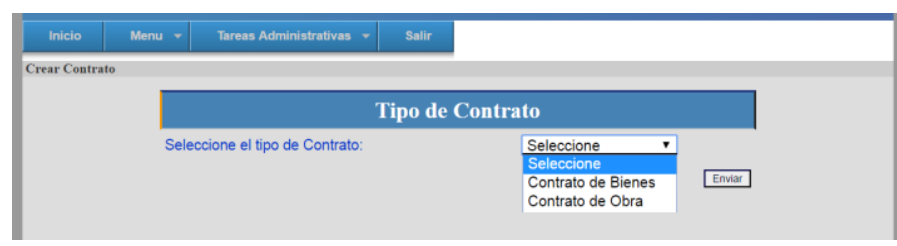

Figura 7. Pantalla de Selección de tipo de Contrato.

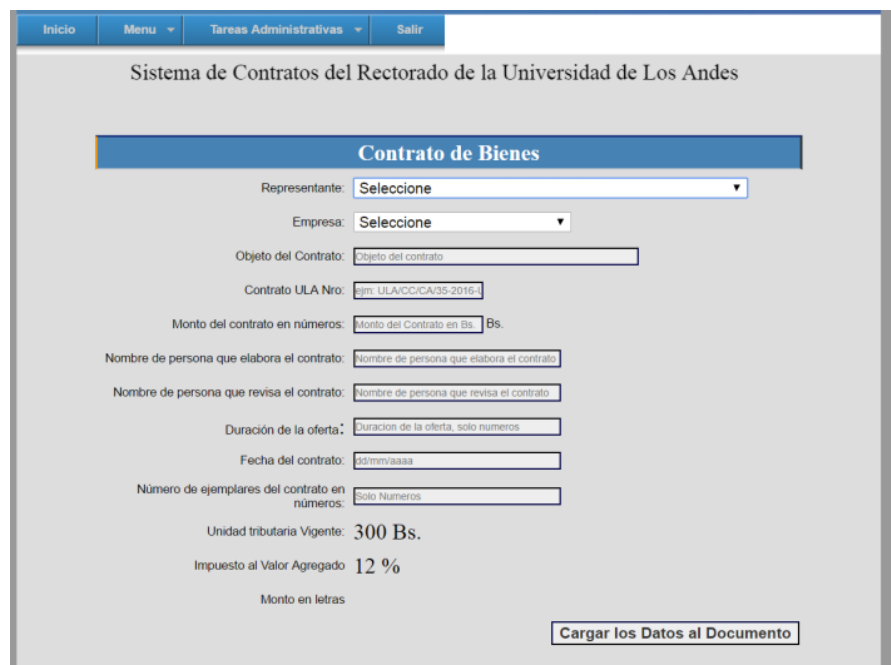

Figura 8. Pantalla de Suministro de Datos para creación de contratos.

A partir de toda la información suministrada, es creado de forma automática el contrato que incluye todas las cláusulas requeridas y posee la información asociada al tipo de contrato, marco tributario, aportes sociales, partes involucradas, y todos los demás elementos subjetivos, objetivos y jurídicos.

\section{CONCLUSIONES}

Con el desarrollo de este sistema se determinó que la técnica de inteligencia artificial que puede ser apropiada para el desarrollo de un sistema automático para la creación de los contratos, son los sistemas basados en conocimiento, ya que utilizan el conocimiento manejado a través de la ley y de las características generales del contrato manejado por el abogado experto y con el cual se puede adaptar los requerimientos del sistema de forma fácil y ajustada a las necesidades.

Se determina que una aplicación web con creación de usuarios y manejo de base de datos para el almacenamiento de datos de empresas e información de los contratos, es la ideal para que los abogados puedan hacer uso del sistema desde diferentes lugares y utilizando diversos sistemas operativos sin problema. Se logró hacer la implementación de una primera versión del sistema, el cual cumple con todas las características deseadas en el diseño.

Se logró hacer la implementación para los contratos de adquisición de bienes y ejecución de obras; se recomienda seguir trabajando con los contratos de prestación de servicios dado que por la diversidad de actividades que pueden estar involucradas en este tipo de contratos, amerita una revisión exhaustiva para verificar la posibilidad de crear un formato genérico para su gestión.

Dado que se logró realizar la implementación de una versión completamente operativa del sistema, la misma debe someterse a pruebas y validaciones por parte de los abogados de las diferentes dependencias que realizan los contratos y determinar posibles mejoras a ser incorporadas.

Es necesario destacar la importancia que tiene la experiencia en la creación de los contratos y que poder realizar un sistema que recopile esa experticia puede ser de utilidad para las organizaciones que suscriben los contratos.

Se debe destacar la importancia del desarrollo de actividades de carácter inter, multi y transdisciplinario en el aporte de soluciones a problemas que puede ser de importancia para las organizaciones. En este caso el uso sinérgico de conceptos del Derecho, de la Ingeniería y de la Inteligencia Artificial proporcionan una solución que ayuda a los abogados encargados de la creación de los contratos en el marco de la Ley de Contrataciones Públicas. Esta ayuda está vinculada al tiempo de redacción del contrato, unificación de criterios asociados a los formatos a ser utilizados, sencillez, seguridad y minimización de errores, entre otros.

\section{REFERENCIAS}

Aguilar, J., y Rivas F. (2001). Introducción a las técnicas de computación inteligente. Mérida, Venezuela: Editorial Meritec.

Akerkar, A.R., y Sajja, P. (2009). Knowledge-Based Systems. Massachusetts, Estados Unidos: Jones \& Bartlett Publishers.

Asprino, M. (2016). Sesión 1. La Administración Pública en el sentido subjetivo y orgánico. Derecho Administrativo II. Mérida, Venezuela: Centro de Estudios Interactivos a Distancia.

Benchimol, G., Levine, P., y Pomerol, J. (1988). Los sistemas expertos en la empresa. Madrid, España: Editorial Ra-Ma.

Bravo, C.E., Saputelli, L., Rivas, F. Pérez, A.G., Nickolaou, M., Zangl, G., De Guzmán, N., Mohaghegh S.D., y Nunez, G. (2014). State of the Art of Artificial Intelligence and Predictive Analytics in the E\&P Industry: A Technology Survey. SPE Journal. 19(4). 547-563.

Congreso de la República de Venezuela (1982). Código Civil de Venezuela. Obtenido

https://derechovenezolano.files.wordpress.com/2012/08/codigo-civilvenezolano.pdf_(Marzo, 2019)

Delgado, M. (2019). La necesidad de la planificación de la contratación como garantía de transparencia, del uso estratégico de la contratación pública y del uso adecuado de los procedimientos de contratación. Gabilex. Revista del Gabinete Jurídico de Castilla-La Mancha. (2). 545-566.

García, A. (2012). Sesión 3. Análisis funcional y estructural de la relación obligatoria. Derecho Civil III (Obligaciones). Mérida, Venezuela: Centro de Estudios Interactivos a Distancia.

García Serrano, A. (2012) Inteligencia Artificial: Fundamentos, práctica y aplicaciones. San Fernando de Henares, España: RC Libros.

Hernández, J. (2008). El contrato administrativo en la Ley de Contrataciones Públicas venezolana. Revista de Administración Pública. (176), 363-381.

Hidalgo, L. (1997). Inteligencia Artificial y Sistemas Expertos. Córdoba, España: Universidad de Córdoba.

Organización Mundial del Comercio (n.d.) Panorama general de la labor de la $\mathrm{OMC}$ en materia de contratación pública. Obtenido de https://www.wto.org/spanish/tratop_s/gproc_s/overview_s.htm__ (Junio 2019)

Presidencia de la República Bolivariana de Venezuela (2008). Ley de Contrataciones Públicas. Gaceta Oficial de la República Bolivariana de Venezuela Nro 38.895. Obtenido de http://www.oas.org/juridico/spanish/mesicic3_ven_anexo20.pdf (Marzo 2019)

Presidencia de la República Bolivariana de Venezuela (2014). Decreto con Rango, Valor y Fuerza de Ley de Contrataciones Públicas. Gaceta Oficial de la República Bolivariana de Venezuela Nro 6.154. Obtenido de www.snc.gob.ve/file/267/download?token=bqaBSRxg (Marzo 2019)

Rivas-Pérez, F., Dos Santos, A., Pardo, V., Rivas-Echeverría, F. (2013). Expert System for appropriate actions to be taken before, during and after a Seismic event in Venezuela. International Journal of Geology. 3 (7), 80-87. 
Rivas F., Colina E., Rivas C. (1998). Expert Systems methodology for Management. Proceeding of IASTED International Conference on Software Engineering. Las Vegas, USA.

Rivas, F. y Arciniegas, S. (2016). Avances y Aplicaciones de Sistemas Inteligentes y Nuevas Tecnologías. Mérida, Venezuela: Consejo de Publicaciones, Universidad de Los Andes.

Rodríguez-Ferrara, M. (2002). Introducción al Derecho de Obligaciones. Obtenido de http://webdelprofesor.ula.ve/cjuridicas/mauricio/capi1.htm (Mayo 2019)

Servicio Jurídico de la Universidad de Los Andes (n.d.). Portal del Servicio Jurídico de la ULA. Obtenido de http://www2.ula.ve/cja/index.php?option=com_frontpage \&Itemid=1 (Marzo 2019)

Stuart, R. y Norvig, P. (2009). Artificial Intelligence: A Modern Approach 3rd Edition. New York, USA: Pearson.

Universidad de Los Andes (n.d.). ¿Qué es la ULA?. Obtenido de http://www.ula.ve/institucion/la-universidad/la-universidad-de-los-andes (Marzo 2019)

\section{BIOGRAFÍAS}

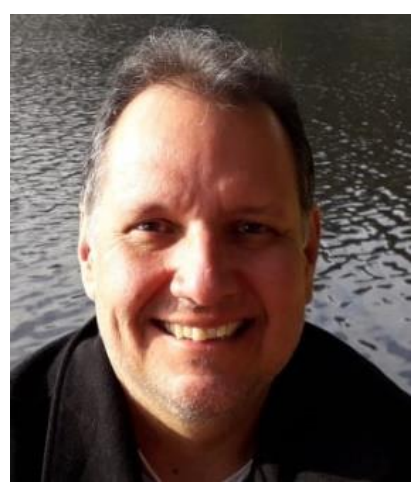

Francklin Rivas Echeverría: recibió el título de Ingeniero de Sistemas de la Universidad de Los Andes (ULA) en 1993. Recibió el grado de Magister Scientiae en Ingeniería de Control en 1996, el Grado de Doctor en Ciencias Aplicadas en 2000 y el título de Abogado (Distinción Cum Laude) en 2017. Es Académico del Departamento de Informática de la Universidad Técnica Federico Santa María, Profesor Titular Jubilado de la Escuela de Ingeniería de Sistemas de la Universidad de Los Andes. Docente de la Pontificia Universidad Católica del Ecuador, Sede Ibarra e Investigador Prometeo (Ecuador). Ha sido Profesor invitado en varias universidades de diversos países, presidente de consorcios internacionales y editor o miembro de consejos editoriales de varias revistas. Asesor de la Organización de las Naciones Unidas, Kuwait Oil Company, Halliburton, entre otros. Ha dirigido más de 80 Proyectos o tesis de grado a nivel de licenciatura, maestría y doctorado. Ha publicado más de 280 trabajos en revistas, memorias de congresos y capítulos de libro. Coautor o editor de 14 libros. Reconocimiento entregado por la Empresa Halliburton por "contribuciones y dedicación al desarrollo de la Tecnología petrolera". Reconocimiento por la Revista Internacional "Gerente" por estar entre los 100 Gerentes Venezolanos más exitosos.

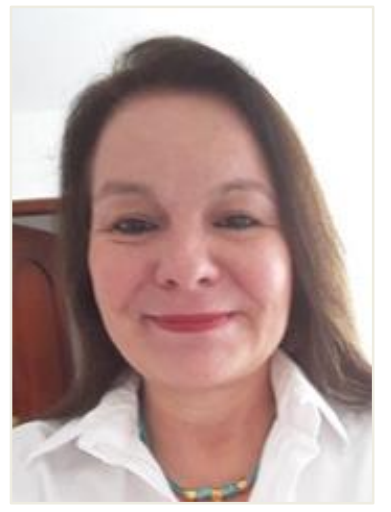

Marilena Asprino Salas: es abogada egresada de la Universidad de Los Andes (ULA) en 1986. Recibió el grado de Magister Scientiae en Derecho Agrario (Instituto Iberoamericano de Derecho Agrario y Reforma Agraria) en el año 1.991; Especialista en Propiedad Intelectual (EPI) en el año 1997 y Doctora en Derecho por la Universidad de Salamanca, España (2007). Profesora titular jubilada de la Facultad de Ciencias Jurídicas, Políticas y
Criminológicas de la Universidad de Los Andes (1991-2016). Dirigió el Centro de Investigaciones Jurídicas y fue Editora Jefe de la Revista Anuario de Derecho (ULA) de 2006 a 2011. Miembro fundador del Grupo de Investigación sobre Políticas Públicas en Propiedad Intelectual (G3PI); investigadora colaboradora del Grupo de Investigación sobre Asuntos Indígenas (GTAI) y Coordinadora del Grupo de Estudios Sociojurídicos (GESJUR) de la Universidad de Los Andes. Fue Coordinadora de Investigación de la UNIANDES, sede Tulcán (Ecuador), 2017. Es docente e investigadora y Coordinadora Académica de la carrera de Derecho en la Escuela de Jurisprudencia de la Pontificia Universidad Católica del Ecuador sede Ibarra (PUCESI). Directora del Grupo de Investigación Derechos Humanos y Control Social (DEHUCS). Autora y coautora de publicaciones científicas, entre libros, capítulos de libros y artículos científicos.

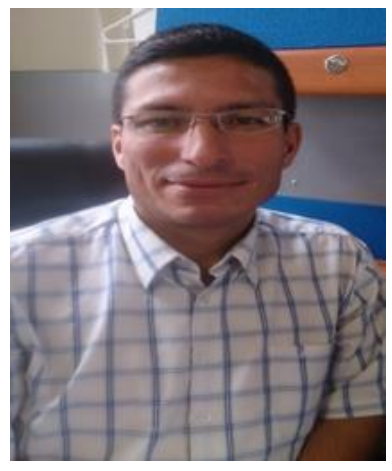

Juan Carlos Sarache Balza: Abogado Jefe de la Dirección de los Servicios Jurídicos de la Universidad de Los Andes. Egresado de esta Universidad, recibió el Título de Abogado en julio del año 2007, mención CUM LAUDE. Posee la Escolaridad en la Especialización en Derecho Administrativo culminada y en proceso para defensa del trabajo especial de grado. Coordina el área jurisdiccional en todos los asuntos judiciales en que la Universidad es parte, incluyendo los procesos en casación y de revisión constitucional. Como miembro Principal de la Comisión Central de Contrataciones Públicas de la Universidad de Los Andes, asiste a la comisión en todos los procesos en las distintas modalidades de selección de contratitas y proveedores, supervisando al mismo tiempo, todos los tramites jurídico-legales de los mismos. Profesor invitado en la Cátedra de Derecho Administrativo de la Facultad de Ciencias Jurídicas y Políticas de la Universidad de Los Andes es Poseedor de varias distinciones honorificas entre las que se destacan las ordenes Ciudad de Mérida, Ciudadano Meritorio y Cruz de las Fuerzas Armadas Policiales del Estado Mérida.

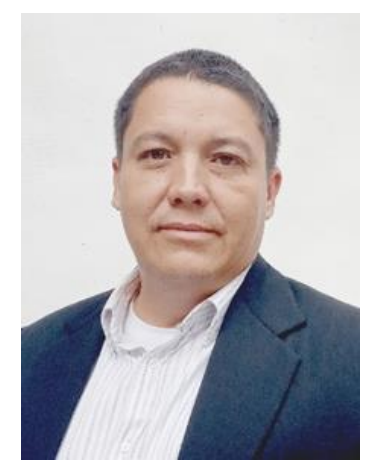

Francisco José León Díaz: Ingeniero de Sistemas de la Universidad de Los Andes (ULA) en 2001, grado de Magister Scientiae en Educación Mención Informática y Diseño Instruccional (ULA) 2012. Especialista de Tecnología de Información Y Comunicación, adscrito al Rectorado de la Universidad de Los Andes, Profesor invitado de la Maestría en Educación Mención Informática y Diseño Instruccional de la Facultad de Humanidades y Educación de la Universidad de Los Andes. 